\title{
Harnessing Wnt signaling as a targetable therapy in epithelial ovarian cancer
}

\author{
Jaclyn A. Wall, Rebecca C. Arend \\ Division of Gynecologic Oncology, Department of Obstetrics and Gynecology, University of Alabama at Birmingham, Birmingham, AL, USA \\ Correspondence to: Rebecca C. Arend, MD. University of Alabama at Birmingham, $61919^{\text {th }}$ Street South, 176F Room 10250, Birmingham, AL, USA. \\ Email: rarend@uabmc.edu. \\ Provenance and Peer Review: This article was commissioned and reviewed by the Executive Editor Dr. Zhi-De Hu (Department of Laboratory \\ Medicine, the Affiliated Hospital of Inner Mongolia Medical University, Hohhot, China). \\ Comment on: Moore KN, Gunderson CC, Sabbatini P, et al. A phase 1b dose escalation study of ipafricept (OMP54F28) in combination with \\ paclitaxel and carboplatin in patients with recurrent platinum-sensitive ovarian cancer. Gynecol Oncol 2019;154:294-301.
}

Submitted Jan 11, 2020. Accepted for publication Feb 19, 2020.

doi: $10.21037 /$ atm.2020.03.16

View this article at: http://dx.doi.org/10.21037/atm.2020.03.16

Epithelial ovarian cancer (EOC) is the deadliest of gynecologic malignancies and the majority of patients are diagnosed at an advanced stage. The 5-year survival for women with stage IIIC EOC, which is the most common stage at the time of diagnosis, remains less than $50 \%$ (1) and the 5 -year relative survival for all advanced stage EOC is approximately $30 \%$ (2). Even following a complete clinical response to initial chemotherapy in conjunction with optimal surgical cytoreduction, the vast majority of these patients will experience a recurrence, thus necessitating continued investigation of beneficial therapies and consideration of novel approaches. Chemotherapy with a platinum/taxane doublet has long been the standard of care for treatment of EOC; this regimen is used in current practice as the typical choice for first-line chemotherapy and for the treatment of recurrent disease when the progression-free interval is at least six months. Following this regimen, various options exist for treating recurrent disease, though there is no pure "gold standard" due to largely inconsistent responses to treatment between patients. In relatively recent years, the angiogenesis inhibitor bevacizumab and poly (ADP-ribose polymerase) (PARP) inhibitors have been approved for maintenance therapies in select groups of patients with EOC; while PARP inhibitors are most efficacious in patients with either somatic or germline $B R C A$ mutations, as of yet there are no predicative biomarkers for which patients may derive the most benefit from bevacizumab maintenance therapy (3).
Thus, while these therapies offer promising options in select groups of patients, a treatment gap persists for many women with recurrent or progressive disease.

Wnt signaling is markedly complex and has roles in human development and organogenesis in addition to its implication in certain cancers. Alterations in the Wnt signaling pathway have been identified in multiple cancer subtypes, including EOC (4), and are associated with promoting tumor growth. Ongoing studies investigating the efficacy of targeting the Wnt pathway have shown promising results in patients harboring Wnt signaling mutations in various cancers $(5,6)$. While the exact mechanism of how Wnt signaling alterations contribute to tumorigenesis is unknown, it is postulated that targeting Wnt may have effects on tumor immunogenicity in addition to direct cell cytotoxicity (7). As immunotherapies overall have seen modest success in EOC, developing new therapies that can harness the host's immune system as another route of attack would open new avenues of treatment for many patients.

Ipafricept (IPA) is a recombinant protein that serves as a Wnt inhibitor through blocking the interaction of Frizzled (FZD) with the FZD8 receptor $(8,9)$, a necessary component in the Wnt signaling pathway. Previous work in mouse models demonstrated that IPA was associated with a decrease in specific cell populations with the ability to reconstitute tumor cells (akin to cancer stem cells). Additionally, results from a xenograft model of EOC 
suggested that a combined therapeutic approach of IPA with a taxane-containing regimen in a sequential rather than a concurrent fashion had superior efficacy (10). Moore and colleagues report the results of a phase $1 \mathrm{~b}$ study of IPA (OMB-54F28) in combination with carboplatin and paclitaxel in recurrent platinum-sensitive EOC.

The primary objectives in this study were to determine the safety and tolerability of IPA in combination with carboplatin and paclitaxel, dose-limiting toxicities (DLT), the maximum tolerated dose (MTD), and the recommended phase 2 dosing regimen. Secondary objectives included characterization of the drug's pharmokinetic profile, immunogenicity, and clinical activity in combination with carboplatin/paclitaxel. Importantly, during the dose escalation phase, IPA was discontinued in two cohorts (those receiving IPA at 5 and $10 \mathrm{mg} / \mathrm{kg} \mathrm{q} 3 \mathrm{w}$ ) as fragility fractures were observed in phase 1 programs of IPA as well as vantictumab, an antibody that inhibits Wnt signaling by binding to various FZD receptors. The remaining cohorts received IPA at lower doses $(2,4$, and $6 \mathrm{mg} / \mathrm{kg} \mathrm{q} 3 \mathrm{w})$ and carboplatin and paclitaxel were administered at AUC $=5 \mathrm{mg} / \mathrm{mL}^{*} \min$ and $175 \mathrm{mg} / \mathrm{m}^{2}$, respectively on day 3 of each cycle (as opposed to day 1 in cohorts 1 and 2) due to the superior efficacy of this regimen in prior studies as described above.

In this study, no DLTs were identified. The MTD was not determined as following implementation of the revised bone safety plan no patients experienced any treatment related adverse events (TRAE) qualifying as a DLT or fragility fracture. Notably, however, all serum bone turnover markers decreased compared to baseline levels. Consequently, the study was prematurely discontinued and the development of IPA was ceased due to the incidence of fragility fractures in both IPA $(6 \%)$ and vantictumab (12\%) programs (11); prior to study discontinuation, $75.7 \%$ of enrolled patients had either a complete or partial response.

As mentioned, there is an unmet need for further therapeutic options in recurrent platinum-sensitive ovarian cancer, specifically targeted therapies, as well as therapies harnessing the host's immune system. This need is likely to further increase due to the fact that EOC patients will likely be receiving maintenance therapy in the upfront setting, particularly with PARP inhibitors, which will increase the population of patients with platinum-sensitive ovarian cancer. Targeted therapies have begun to infiltrate the oncology landscape in recent years and have seen success in many instances. Within EOC specifically, the introduction of PARP inhibitors has arguably yielded the most success and possibly had the most immediate effect on treatment practices, but as only roughly $15 \%$ of EOC patients harbor $B R C A$ mutations (12), identifying other targetable pathways is highly indicated. Moore and colleagues provide compelling evidence that targeting Wnt signaling may prove to have notable clinical efficacy in such patients given an overall response rate of $75.7 \%$ in their study, though they did not include specific testing for Wnt target mutations. The discontinuation of IPA and vantictumab due to poor bone safety profiles should not damper investigation into other Wnt-targeting therapies. Currently, there is an ongoing phase 2 clinical trial investigating DKN-01, a monoclonal antibody targeting Dickkopf-1 (DKK1), as monotherapy or in combination with paclitaxel in advanced gynecologic malignancies, including EOC (NCT03395080). DKK1 itself, via a negative feedback loop, inhibits the Wnt pathway, particularly in the setting of Wnt upregulation; however, despite its role in negative feedback, increased DKK1 levels are poorly prognostic in several cancers, including ovarian cancer (13), and in nonclinical models increased DKK1 has been shown to promote cancer cell migration, tumor growth, metastasis and angiogenesis and may play an immune-modulatory role.

Moving forward, the Wnt pathway remains a promising therapeutic target in advanced ovarian cancers and has gained increased enthusiasm lately given its potential role in immune evasion. While IPA development has been discontinued due to a poor bone safety profile, the clinical efficacy reported by Moore and colleagues speaks to the potential utility of targeting Wnt signaling in this patient population. Available clinical and pre-clinical data investigating other modulators of Wnt signaling suggests that perhaps the key to their therapeutic benefit in EOC and other advanced gynecologic cancers lies in an immunomodulatory role. Continued work to unravel the mechanistic underpinnings of how altered Wnt signaling creates an avenue for these cancers to potentially escape immunosurveillance and translate this into an effective anticancer targeted therapy is highly warranted.

\section{Acknowledgments}

Funding: None.

\section{Footnote}

Conflicts of Interest: RCA: Advisory board, Clovis, Tesaro, AstraZeneca, Leap. JW has no conflicts of interest to declare. 
Ethical Statement: The authors are accountable for all aspects of the work in ensuring that questions related to the accuracy or integrity of any part of the work are appropriately investigated and resolved.

Open Access Statement: This is an Open Access article distributed in accordance with the Creative Commons Attribution-NonCommercial-NoDerivs 4.0 International License (CC BY-NC-ND 4.0), which permits the noncommercial replication and distribution of the article with the strict proviso that no changes or edits are made and the original work is properly cited (including links to both the formal publication through the relevant DOI and the license). See: https://creativecommons.org/licenses/by-nc-nd/4.0/.

\section{References}

1. Webb PM, Jordan SJ. Epidemiology of epithelial ovarian cancer. Best Pract Res Clin Obstet Gynaecol 2017;41:3-14.

2. NCI. SEER 18 Registries. Available online: https://seer. cancer.gov/

3. Moore KN, Gunderson CC, Sabbatini P, et al. A phase $1 \mathrm{~b}$ dose escalation study of ipafricept (OMP54F28) in combination with paclitaxel and carboplatin in patients with recurrent platinum-sensitive ovarian cancer. Gynecol Oncol 2019;154:294-301.

4. Luke JJ, Bao R, Sweis RF, et al. WNT/beta-catenin Pathway Activation Correlates with Immune Exclusion across Human Cancers. Clin Cancer Res 2019;25:3074-83.

5. Arend RC, Castro CM, Matulonis UA, et al. Safety and Efficacy of a DKK1 Inhibitor (DKN-01) as Monotherapy or in Combination with Paclitaxel in Patients with Wnt Activated Recurrent Gynecologic Malignancies. Gynecol

Cite this article as: Wall JA, Arend RC. Harnessing Wnt signaling as a targetable therapy in epithelial ovarian cancer. Ann Transl Med 2020;8(13):837. doi: 10.21037/atm.2020.03.16
Oncol 2019;154:34.

6. Edenfield WJ, Richsrds DA, Vukelja SJ, et al. A phase 1 study evaluating the safety and efficacy of DKN-01, an investigational monoclonal antibody (Mab) in patients (pts) with advanced non-small cell lung cancer. J Clin Oncol 2014;32:8068.

7. Kagey MNG, Haas M, Heath $\mathrm{H}$, et al. High tumor expression of DKK1 is associated with improved clinical benefit and longer progression free survival across multiple solid tumors when treated with a target anti-DKK1 antibody (DKN-01). [Poster].

8. Le PN, McDermott JD, Jimeno A. Targeting the Wnt pathway in human cancers: therapeutic targeting with a focus on OMP-54F28. Pharmacol Ther 2015;146:1-11.

9. Katoh M, Katoh M. Molecular genetics and targeted therapy of WNT-related human diseases (Review). Int J Mol Med 2017;40:587-606.

10. Fischer MM, Cancilla B, Yeung VP, et al. WNT antagonists exhibit unique combinatorial antitumor activity with taxanes by potentiating mitotic cell death. Sci Adv 2017;3:e1700090.

11. Kurzeder C, Bover I, Marmé F, et al. Double-Blind, Placebo-Controlled, Randomized Phase III Trial Evaluating Pertuzumab Combined With Chemotherapy for Low Tumor Human Epidermal Growth Factor Receptor 3 mRNA-Expressing Platinum-Resistant Ovarian Cancer (PENELOPE). J Clin Oncol 2016;34:2516-25.

12. Norquist BM, Harrell MI, Brady MF, et al. Inherited Mutations in Women With Ovarian Carcinoma. JAMA Oncol 2016;2:482-90.

13. Kagey $M H, H e X$. Rationale for targeting the Wnt signalling modulator Dickkopf-1 for oncology. $\mathrm{Br} \mathrm{J}$ Pharmacol 2017;174:4637-50. 